\title{
Implementação de intervenções breves para uso problemático de álcool na atenção primária, em um contexto amazônico
}

\author{
Rodrigo Otávio Moretti-Pires ${ }^{1}$ \\ Clarissa Mendonça Corradi-Webster ${ }^{2}$
}

O objetivo do estudo foi avaliar o processo de implementação de estratégias de rastreamento e intervenções breves para o uso problemático de álcool (UPA), na atenção primária, do município de Coari, Amazonas. A metodologia usada nesta pesquisa foi do tipo avaliação para gestão por análise do processo, através da triangulação de métodos, utilizando-se técnicas qualitativas, como grupos focais com profissionais de saúde e equipe envolvida na capacitação/monitorizaçã, e dados quantitativos epidemiológicos e de produtividade, em serviços de atenção do ano 2008. Como resultados, obteve-se que o treinamento foi bem aceito pela equipe, que se sentiu valorizada. Da amostra, $25 \%$ pontuou UPA no Alcohol Use Disorders Identification Test (AUDIT). As dificuldades apresentadas se referem à preservação do enfoque biomédico, ainda, dificuldade para dar continuidade ao trabalho devido à rotatividade dos profissionais por motivos políticos e para estabelecer políticas para áreas de pouco acesso. Conclui-se que há necessidade de ações de gestão e de políticas de saúde, direcionadas à temática do UPA, na atenção primária, sobressaindo a necessidade de fixação do recurso humano das equipes de Estratégia de Saúde da Família.

Descritores: Estudos de Avaliação; Atenção Primária à Saúde; Alcoolismo.

\footnotetext{
${ }^{1}$ Professor Doutor, Centro de Ciências da Saúde, Universidade Federal de Santa Catarina, Florianópolis, Brasil. E-mail: rodrigo.moretti@pq.cnpq.br.

2 Psicóloga, Doutora em Psicologia, Escola de Enfermagem de Ribeirão Preto, Universidade de São Paulo, Centro Colaborador da OMS para o Desenvolvimento da Pesquisa em Enfermagem, SP, Brasil. E-mail: clarissac@usp.br.
}

Correspondencia:

Clarissa Mendonça Corradi-Webster

Universidade de São Paulo. Escola de Enfermagem de Ribeirão Preto

Departamento de Enfermagem Psiquiátrica e Ciências Humanas

Av. dos Bandeirantes, 3900

Bairro: Monte Alegre

CEP: 14040-902 Ribeirão Preto, SP, Brasil

E-mail: clarissac@usp.br 


\title{
Implementación de las intervenciones breves para casos de uso problemático de alcohol en el la atención primaria en un contexto amazónico
}

El objetivo de este estudio fue evaluar el proceso de implementación de las estrategias de rastreo e intervenciones breves para casos de uso problemático de alcohol (UPA) en la atención primaria en el municipio de Coari, Amazonas. Se trata de una investigación para la evaluación del tipo de examen de la gestión por proceso, a través de la triangulación de métodos, utilizando grupos focales con profesionales e investigadores que participan en la formación y seguimiento; utilizando datos epidemiológico y de productividad en los servicios de atención. La capacitación fue bien aceptada por el equipo. El 25\% puntuaron el UPA en el AUDIT. Fueron detectados como problemas: la preservación del enfoque biomédico, la continuidad de la labor debido a la rotación de profesionales y el establecimiento de políticas para las zonas de acceso limitado. Se concluye que existe una necesidad de implementar medidas de gestión y políticas dirigidas a la UPA en la AP, destacándose la necesidad de determinar recursos humanos para los equipos de AP.

Descriptores: Estudios de Evaluación; Atención Primaria de Salud; Alcoholismo.

\section{Implementation of Brief Intervention for Problematic Alcohol Use in Primary Health in the Amazon Context}

\begin{abstract}
Aims: to evaluate the implementation process of Screening and Brief Interventions for the Problematic Use of Alcohol (PUA) in primary health care in the city of Coari, Amazonas. Methods: management evaluation through a process analysis study, using triangulation methods, with qualitative techniques like focus groups with health professionals and with the training / follow up team; quantitative epidemiological data and data concerning health service productivity in 2008. Results: the health team accepted the training course well, feeling that it valued them. Twenty-five percent of the sample scored PUA using AUDIT. Difficulties: preservation of the biomedical approach, difficulties to continue the work because of high professional rotation levels due to political reasons, difficulties to stabilize policies in places with little access. Conclusions: management action is needed, as well as health policies for PUA in the PHC, including the need for fixed human resources in $\mathrm{PHC}$ teams.
\end{abstract}

Descriptors: Evaluation Studies; Primary Health Care; Alcoholism.

\section{Introdução}

A adoção da Estratégia de Saúde da Família (ESF), como principal configuração da atenção primária (AP) no Brasil, baseia-se no discurso que prima por romper com o modelo biomédico e fragmentado em especialidades. Nesse sentido, há clara necessidade de articulação entre suas ações e o campo da saúde mental (SM), que deve ser entendido como temática transversal e pertinente a todos os níveis de atenção, valorizando a promoção e proteção específica na área, tanto quanto os aspectos curativos em rede de serviços ${ }^{(1)}$.

Dada a imersão dos profissionais de saúde na dinâmica de vida dos usuários, de suas famílias e de seus entornos socioculturais, as equipes da ESF têm importância estratégica para o enfrentamento de temáticas de SM, tais como o uso problemático de substâncias psicoativas e as diversas modalidades de sofrimento psíquico(2). No entanto, essas mesmas equipes ainda encontram dificuldades para lidar com essas dimensões, apesar da crescente valorização da dimensão subjetiva dos usuários e a monitorização/ enfrentamento dos problemas mais frequentes de SM, nas Políticas Públicas de AP, no Brasil ${ }^{(2-4)}$. 
O uso do álcool é questão relevante dentro dos problemas de Saúde Pública da atualidade, estando relacionado a diversos problemas sociais, causas de adoecimento e comorbidades(5). Os dados no Brasil indicam que $23 \%$ dos adultos já beberam e tiveram problemas com álcool, $28 \%$ já beberam de forma abusiva, em pelo menos uma ocasião, no período de 12 meses, 20\% desses bebedores apresentam frequência maior do que semanal, sendo que $20 \%$ em suas próprias residências $^{(6)}$. Os dados ainda apresentam que 3\% dos brasileiros fazem uso nocivo e $9 \%$ são dependentes de bebidas alcoólicas(6).

Dada a relevância da temática, o discurso estatal contemporâneo prima por ações de prevenção primária, diagnóstico precoce, redução de danos e tratamento de casos não complicados para as pessoas que fazem uso problemático da substância e suas famílias, no âmbito da ESF, articulada à rede de assistência em $\mathrm{SM}^{(3)}$, destacando-se a importância de estratégias que permitam o rastreamento dos problemas com álcool na $A P$ e a intervenção nos níveis de prevenção que competem às equipes da ESF.

O principal instrumento, atualmente, para rastreamento de problemas relacionados ao consumo de bebidas alcoólicas é o A/cohol Use Disorders Identification Test (AUDIT) ${ }^{(7)}$, cuja importância, nesse campo de SM em $A P$, se dá por: (1) ser de fácil utilização por todos os profissionais desde que devidamente capacitados, (2) estar atrelado a intervenções breves direcionadas aos padrões de utilização da substância, que são contínuos e fogem à lógica reducionista da "dependência/não dependência", permitindo que o profissional intervenha em termos da vulnerabilidade e do risco, aos quais está exposto o usuário e (3) por ser composto por dez questões, o tempo utilizado para o rastreamento e intervenção varia de cinco a dez minutos, não prejudicando o processo de cuidado tradicional na ESF.

De acordo com a pontuação do AUDIT, o profissional identifica quatro zonas de risco, sendo essas: 1 . uso de baixo risco (consumo dentro dos limites considerados pela OMS como 'seguros'), 2. uso de risco (consumo acima dos limites, entretanto, sem apresentação de problemas), 3. uso nocivo (consumo acima dos limites, tendo resultado em problemas) e 4. provável dependência. Após a identificação do padrão de consumo, o profissional está instrumentalizado para realizar as orientações que têm, como características principais, o fato de serem breves (2 a 10 minutos) e, a partir do feedback dado pelo profissional ao paciente a respeito de sua pontuação no instrumento, de buscar motivá-lo a mudar seu comportamento. Esse tipo de intervenção vem sendo conhecida como "intervenções breves" (IB) e podem ser realizadas por toda a equipe da ESF, desde que tenham recebido treinamento para a condução dessa(7). A literatura vem demonstrando que a AP é espaço importante para o uso das IB, já que privilegia as ações de promoção e prevenção. Nesse sentido, diversos estudos mostram que as IB auxiliam na redução do consumo de bebidas alcoólicas, principalmente entre as pessoas que fazem uso de risco( $^{(7)}$.

Diante do exposto, o presente artigo tem por objetivo avaliar o processo de implementação de estratégias de rastreamento e intervenções breves (ERIB) para uso problemático de álcool na $\mathrm{AP}$, no contexto amazônico do município de Coari, interior do Estado do Amazonas.

\section{Metodologia}

O estudo foi desenvolvido no município de Coari, interior do Estado do Amazonas, localizado à margem direita do Rio Solimões, com distância, em relação à Manaus, de $363 \mathrm{~km}$ em linha reta e $463 \mathrm{~km}$ por via fluvial. Sua área territorial é de $57.230 \mathrm{~km}^{2}$, clima quente e úmido, com período chuvoso nos meses de novembro a fevereiro. A temperatura média situa-se entre 31 e $35^{\circ} \mathrm{C}$. A população de Coari é de 81.053 habitantes, estratificada entre 48.053 habitantes que residem na zona urbana e 33.041 na zona rural, incluindo, nessa última, a população das comunidades ribeirinhas, composta por 250 comunidades que variam entre 20 e 400 habitantes entre crianças, adultos e idosos.

Trata-se de pesquisa avaliativa, do tipo avaliação para gestão, priorizando a análise do processo de implementação de ações, através triangulação de métodos $^{(8)}$, norteando-se pela avaliação de três momentos: (1) o contexto prévio à implementação, (2) o processo de implementação propriamente dito e (3) os resultados da implementação, junto às equipes de ESF.

Para se avaliar o contexto prévio, foram analisados os recursos disponíveis, buscando-se caracterizar a rede de atenção à SM disponível para os usuários das ESFs, entrevistando o gestor da Secretaria Municipal de Saúde, quanto à organização da rede municipal, a respeito de questões de referência e contrareferência dos casos pertinentes, além de acompanhamento da produção em SM do município, no ano 2008. Em termos do recurso humano envolvido, foram realizados grupos focais com os profissionais da atenção, identificando o conhecimento e visibilidade da temática no cotidiano das ESFs, dados pertinentes aos serviços de saúde. 
O material dos grupos focais foi analisado no quadro conceitual que fundamenta a AP e a realização da ERIB em seu contexto(7), tomando como eixo a identificação da temática no cotidiano da assistência, a capacitação dos profissionais para o enfrentamento do tema, os desafios a serem superados, as estratégias utilizadas durante o processo de implementação e o impacto da implementação no cotidiano da ESF.

O processo de implementação se desenvolveu nas seguintes etapas:

(1) divulgação do projeto através de simpósio sobre a temática, aberto a toda a população e profissionais;

(2) capacitação dos profissionais das ESFs;

(3) implementação inicial do uso do AUDIT na rotina das ESFs, por um mês, sendo realizado levantamento epidemiológico utilizando o AUDIT pelos agentes comunitários de saúde (ACS), durante as visitas domiciliares, com amostra de 667 usuários dos serviços, aleatoriamente selecionados entre a população cadastrada nas ESFs, cujos dados foram tratados utilizando-se o programa estatístico SPSS, versão 16.0. Aplicou-se o teste Kolmogorov-Simirnov para verificar a normalidade da amostra, utilizando-se, em seguida, testes não-paramétricos, sendo que o teste de MannWhitney foi usado para comparação entre as médias em relação ao sexo e o teste de qui-quadrado para as variáveis categóricas, estabelecido nível de significância de $\mathrm{p}<0,001$;

(4) monitoramento semanal das ações e capacitação contínua de ACSs por seis meses. Como instrumentos para avaliação dessa etapa, foram utilizados materiais produzidos nas reuniões quinzenais com os dez membros da equipe de pesquisa, responsáveis pela capacitação e monitoramento das ações junto às ESFs.

Com relação ao terceiro momento, a avaliação dos resultados da implementação, realizou-se grupos focais com os membros da equipe que participaram do monitoramento, confrontando o processo com os achados iniciais, no sentido de identificar avanços e barreiras. Também foram entrevistados os profissionais ACSs das ESFs que mais se envolveram no processo sobre esses mesmos itens.

O projeto que originou os dados foi desenvolvido com recursos oriundos da Fundação de Amparo a Pesquisa do Estado do Amazonas (Edital PPSUS2006), com aprovação ética do Comitê de Ética em Pesquisa da Universidade Federal do Amazonas, Parecer n0114/2007, seguindo os procedimentos legalmente exigidos na coleta das informações, em especial aos referentes ao termo de consentimento livre e esclarecido.

\section{Resultados}

\section{Avaliação do contexto prévio à implementação}

No ano 2008, o Sistema de Saúde do município de Coari contava com onze unidades de saúde da família, duas unidades fluviais de atenção, um hospital de adscrição regional e um Campus Avançado da Fundação de Medicina Tropical. A equipe de saúde do SUS era composta por 28 médicos, 35 enfermeiros, 14 odontólogos, 2 nutricionistas, 2 psicólogos, 3 assistentes sociais, 3 fisioterapeutas e 223 agentes comunitários de saúde, dos quais 120 (53,8\%) atuavam na zona urbana.

Não existe serviço estruturado de psicologia e/ou psiquiatria, sendo que os dois psicólogos atendem casos no hospital, sem seguimento. A produção realizada em termos de psicologia foi de 1.519 atendimentos no ano 2008 , dado que contrasta com os 5.132 acidentes que foram atendidos no setor hospitalar, sendo que os profissionais apontam que mais da metade desses envolviam pessoas com quadro de intoxicação pelo uso de álcool.

O modelo no qual se estrutura o SUS, em Coari, é a saúde da família, cujo cadastramento abrange $83 \%$ da população urbana (38.765 pessoas, em 7.985 famílias) e $68 \%$ da população rural (21.014 pessoas, em 4.303 famílias), segundo dados do Sistema de Informações de Atenção Básica, fornecidos pela Secretaria Municipal da Saúde de Coari.

Como em toda a realidade amazônica, a questão do acesso é de fundamental importância para o entendimento da realidade da saúde das populações. Seja com fins de atenção à saúde, ou outro, o acesso à sede do município e área rural é realizado apenas por via fluvial ou aérea, não havendo estradas, salvo a sede para algumas comunidades. Para essa importante parcela populacional, os serviços de saúde se restringem ao atendimento curativo na sede do município, ou à atuação de ACSs existentes para cada grupo de comunidades.

Em relação ao conhecimento dos profissionais, tanto nas categorias de profissionais de nível de escolaridade superior como nas categorias técnicas envolvidas na ESF, a visibilidade da temática se dava principalmente em casos de intoxicação, identificando conjuntamente os fatores de risco para o uso problemático de álcool. Outro achado foi o reconhecimento das consequências desse consumo para o indivíduo e para a sociedade, entretanto, não apontaram como atuar sobre essa questão, indicando a necessidade de ações de capacitação no âmbito da ESF. 


\section{Processo de implementação propriamente dito}

Em dezembro de 2007, foi realizado o $1^{\circ}$ Simpósio Amazônico sobre o Uso Problemático de Álcool, tendo como finalidade divulgar a temática e as ações que seriam realizadas no projeto. Os envolvidos na condução dos trabalhos apresentaram um panorama geral da temática e de sua pertinência junto à AP. O evento teve dois dias de duração e o interesse, tanto da população em geral como dos profissionais presentes, foi sobremaneira marcante, tendo como indicadores a frequência durante os dois dias de atividades (312 participantes), inclusive após algumas atividades de debates extrapolarem os horários previstos, invadindo períodos de almoço e jantar, onde o público permaneceu e aprofundava debates.

Na semana seguinte ao Simpósio, foram realizados dois cursos de capacitação no Instituto de Saúde e Biotecnologia da Universidade Federal do Amazonas. Os cursos tiveram 16 horas de duração e foram estruturados de forma gradual, apresentando aspectos epidemiológicos, biológicos, psíquicos e societários envolvidos no consumo de álcool, aspectos de promoção e prevenção em AP, critérios internacionais de classificação, padrões de consumo, AUDIT como instrumento de rastreamento e intervenções breves ${ }^{(7)}$. Nessa ocasião, participaram do treinamento 9 médicos, 7 enfermeiras e 120 agentes comunitários de saúde (ACS) que, em relação à população total de profissionais do município, representaram 32\% dos médicos, 20\% das enfermeiras e $100 \%$ dos ACSs da zona urbana.

Como decorrência, durante o treinamento, foi proposta a implementação inicial do projeto. Para tanto, ficou decidido pelos próprios envolvidos das ESFs que o profissional considerado como ideal para o trabalho seria o ACS. Durante um mês, os ACSs realizaram aplicação do AUDIT e intervenções breves, supervisionados por membros da equipe de pesquisa. Em termos epidemiológicos, a realidade encontrada, junto aos usuários do sistema, foi de $186(25,14 \%)$ pessoas que estavam fazendo uso problemático de álcool, incluindose uso de risco, uso nocivo e provável dependência. Com o uso do teste de Mann-Whitney, foi identificada diferença entre a média de pontos no AUDIT entre homens e mulheres, sendo maior a média de pontos entre os homens $(p<0,001)$. Em relação à porcentagem de homens e mulheres que pontuaram, dentro de cada uma das zonas de risco, observa-se porcentagem de homens maior que de mulheres, nas três zonas, que indicam consumo problemático de álcool (Tabela 1).

Tabela 1 - Porcentagem de indivíduos que pontuaram dentro de cada uma das zonas de risco no AUDIT, de acordo com o sexo $(n=667)$. Coari, 2008

\begin{tabular}{|c|c|c|c|c|}
\hline \multirow[b]{2}{*}{ Sexo* } & \multicolumn{4}{|c|}{ Zona de risco } \\
\hline & $\begin{array}{c}1 \\
\text { (0-7 pontos) }\end{array}$ & $\begin{array}{c}2 \\
(8-15)\end{array}$ & $\begin{array}{c}3 \\
(16-19 \text { pontos) }\end{array}$ & $\begin{array}{c}4 \\
\text { (20-40 pontos) }\end{array}$ \\
\hline Masculino & 57,74 & 22,53 & 6,76 & 12,95 \\
\hline Feminino & 93,92 & 3,51 & 0,95 & 1,27 \\
\hline
\end{tabular}

$* p<0,001$ no teste do qui-quadrado para diferenças nas zonas de risco do AUDIT, em relação ao sexo.

Após essa etapa de implementação prévia, semanalmente, durante seis meses, membros da equipe de pesquisa se encontraram com os ACSs da ESFs, in loco, com o objetivo de monitorar e promover capacitação contínua de ACSs, tanto no sentindo de sanar dúvidas como de encontrar soluções para os desafios que se apresentavam.

\section{Resultados da implementação junto às equipes de ESF}

Durante os grupos focais com membros da equipe do monitoramento, foram descritas algumas barreiras para a implementação do projeto. Uma das principais referiu-se ao processo político eleitoral, vivenciado em 2008, motivo de constantes rodízios de profissionais, demissões e admissões no quadro da ESF, trazendo à equipe de monitoramento a necessidade de iniciar todo o processo de capacitação, junto aos novos profissionais, e a descontinuidade do trabalho em relação àqueles que já haviam sido capacitados.

Outras barreiras descritas foram: a falta de tempo e de treinamento específico durante a graduação/ profissionalização, o medo de possível hostilidade dos pacientes, a percepção de que esse tipo de procedimento não faz parte de suas funções da ESF e a crença de que alcoolistas não respondem ao tipo de intervenção que pode ser dada, no nível primário de atenção. Alguns profissionais de saúde relutaram em examinar e orientar pacientes sobre seus problemas decorrentes do uso do álcool, alegando carga de trabalho excessiva, falta de 
tempo para a equipe atuar com mais precisão, falta de capacitação para esses profissionais atuarem de forma mais direta e precisa na intervenção.

Na avaliação final do projeto, os ACSs entrevistados apontaram que, conforme o AUDIT era aplicado e os dados consolidados, maior importância tomava a temática no trabalho dos mesmos e que, mesmo após o término do projeto, continuariam a discutir a questão do uso problemático da substância em seu dia a dia, na medida em que os dados apontam a pertinência da temática e seu impacto na saúde da população, mas indicaram a falta de motivação dos médicos e de alguns enfermeiros para inserir, na rotina da unidade, as estratégias de rastreamento e intervenções breves, para uso problemático de álcool. Os próprios ACSs denunciaram que os profissionais que não aderiram apresentam discurso pautado na dependência, não conseguindo romper a lógica biomédica, focada na doença, como se apenas a intoxicação e os casos de dependência fossem realmente relevantes para a asssistência, e, na pertinência das temáticas de SM, relevante apenas ao psiquiatra. E como não existe rede de referência, deixaram de lado, por não entenderem como resolver o quadro.

Na percepção da equipe de monitoramento, houve aceitação, empenho e busca do conhecimento por parte dos ACSs no contexto da temática em questão. Muitos ACSs relataram que não sabiam proceder de forma eficaz, quando se deparavam com pacientes com esses tipos de problemas, mas com a utilização do instrumento epidemiológico (AUDIT) para verificar a zona ao qual o paciente se encontrava e as intervenções breves, como forma de conscientização, tornava-se simples proporcionar atendimento correto e eficaz à população. Foi relatado, também, que em momento algum, antes da implantação do estudo, foram-lhes acrescentadas informações referentes ao tema pelos órgãos responsáveis, caracterizando a fragilidade do atendimento à saúde na AP, ficando, assim, explícita a necessidade de ações políticas e de saúde voltadas à temática do uso problemático do álcool. Outra barreira apresentada se refere à assistência à população rural, dados os problemas para acesso a essas comunidades, que se dá somente por via fluvial. Durante o projeto, não foi possível realizar ações nesse grupo, que representa cerca de $40 \%$ da população total.

\section{Discussão}

A exiguidade de ações em SM na ESF é apresentada na literatura científica como indício da necessidade de articulação entre AP e SM, o que foi apontado no processo de avaliação realizado(1). Apesar da boa estrutura do Sistema de Saúde de Coari, AM, com suas onze unidades de saúde da família e hospital de média complexidade, a inexistência de serviço de referência em SM aponta para o silêncio do governo em relação a essas importantes questões, presentes no dia a dia dos profissionais da ESF. O quadro ainda se agudiza em relação à zona rural, assim como aos indicadores de acidentes, relacionados ao uso da substância. A literatura apresenta que essas lacunas entre necessidade e serviços de atenção, mais do que as questões clínicas propriamente ditas, ferem o direito do usuário enquanto cidadão, inclusive porque as questões de SM são igualmente importantes às demais ${ }^{(9-10)}$.

Apesar da pertinência da temática indicada nos depoimentos, ou pelos dados epidemiológicos, a falta de treinamento dos profissionais indica silêncio na sua formação com relação às dimensões subjetivas do ser humano, ferindo princípios da ESF, como a abordagem integral do usuário(11). A falta de treinamento de profissionais para lidar com o consumo problemático de bebidas alcoólicas já vem sendo levantada em outros estudos, necessitando de ações que lidem com essa demanda(12-14). Dado que também chamou a atenção foi a maior prevalência de uso de álcool e padrões problemáticos entre homens, indicando necessidade de ações específicas para essa população que recorre menos aos serviços de saúde, inclusive, achado compatível com outros estudos ${ }^{(15)}$.

O contexto apresentado remete à questão da formação dos profissionais de saúde, que se pauta no modelo biomédico/fragmentário, cuja lógica valoriza as especialidades, havendo coêrencia na relutância/ dificuldade em que os profissionais apresentaram para abordar problemas de saúde de naturezas diferentes da abordagem biologicista(11,15-16). Em outras experiências, semelhantes à presente, foram apontadas dificuldades relacionadas à visão estereotipada em relação à temática(16), apreensão com a abordagem do tema junto aos usuários do sistema e o talhe curativo dentro da $\mathrm{ESF}^{(15)}$.

A eficácia de outras experiências nacionais referiuse, em particular, à sensibilização dos profissionais e da inserção das estratégias de rastreamento, na rotina dos profissionais de $\mathrm{AP}^{(17-18)}$, sendo coerente afirmar que a questão da descontinuidade de recursos humanos nas equipes da ESF investigadas foi a principal barreira à implementação, justamente pela falta de fixação da equipe de profissionais junto aos serviços, prejudicando a continuidade das ações. 
Essas considerações sobre gestão convergem para a literatura que vem defendendo a inserção da SM na $A P^{(1)}$. No que se refere aos usos do AUDIT, existem evidências científicas que recomendam sua adoção, principalmente por sua abordagem no continuum do usuário que é abstêmio ao dependente, permitindo aos profissionais de AP o enfrentamento do problema(19), não apenas os especialistas, mas, sim, todos os profissionais, de forma que se possa implementar monitoramento e execução das ações vinculadas a essa estratégia de abordagem do uso de álcool, nesse nível de atenção, uma vez que as evidências apontam que as intervenções breves, atreladas ao uso do AUDIT, reduzem o consumo do álcool(20).

Neste estudo, o uso de instrumento epidemiológico como forma de sensibilização para ações de saúde, junto aos ACSs, mostrou-se interessante, na medida em que pode se constituir em estratégia para desencadear o processo de educação permanente das equipes.

\section{Conclusões}

As equipes de AP estão em posição privilegiada para identificar e intervir em pacientes cujo consumo de bebidas alcoólicas tornou-se problemático e danoso. Elas podem, também, desempenhar importante papel na motivação e encaminhamento de pacientes dependentes de álcool para tratamento, sendo esse um dos papéis da equipe que atua dentro do setor primário. Porém, o sistema de saúde ainda necessita de reformulação em termos de processo de trabalho, principalmente no que tange aos aspectos de implantação de políticas que se refiram aos aspectos de saúde mental, tais como as estratégias de rastreamento e intervenções breves para uso problemático de álcool.

O uso de instrumento epidemiológico como forma de sensibilização, para ações de saúde junto aos ACSs, se mostrou interessante, na medida em que pode se constituir estratégia para desencadear o processo de educação permanente das equipes.

A proposta de implementação teve adesão significativa dos ACSs, havendo aceitação, empenho e busca do conhecimento por parte dos mesmos, no contexto da temática em questão. Os ACSs se sentiram valorizados por terem a oportunidade de participar de treinamento em saúde, destacando a falta de informações referentes ao tema pelos órgãos responsáveis, caracterizando a fragilidade do atendimento à saúde na $A P$, explicitando a necessidade de ações de gestão e de políticas de saúde, direcionadas à temática do uso problemático do álcool na $\mathrm{AP}$, sobressaindo a necessidade de fixação de recursos humanos nas equipes de ESF.

\section{Agradecimentos}

Agradecemos a Comissão Interamericana para o Controle do Abuso de Drogas/CICAD da Secretaria de Segurança Multidimensional/SSM da Organização dos Estados Americanos/OEA, a Secretaria Nacional de Políticas sobre Drogas/SENAD do Gabinete de Segurança Institucional/Brasil, a Escola de Enfermagem de Ribeirão Preto da Universidade de São Paulo e o Centro Colaborador da Organização Mundial da Saúde para o Desenvolvimento da Pesquisa em Enfermagem, a população representada nas pesquisas, bem como as autoridades das universidades representadas pelos participantes do Programa On-Line de Especialização em Pesquisa sobre o Fenômeno das Drogas - períodos 2006, 2007, 2008 e 2009.

\section{Referências}

1. Amarante PDC. Saúde Mental, desinstitucionalização e novas estratégias de cuidado. In: Giovanella L, Escorel S, Lobato LCV et al, organizadores. Políticas e sistema de saúde no Brasil. Rio de Janeiro (RJ): Editora FIOCRUZ; 2008. p. 735-60.

2. Ministério da Saúde (BR). Reforma psiquiátrica e política de saúde mental no Brasil. Brasília: Ministério da Saúde; 2005.

3. Ministério da Saúde (BR). A Política do Ministério da Saúde para Atenção Integral a Usuários de Álcool e outras Drogas. Brasília: Ministério da Saúde; 2004.

4. Moretti-Pires RO, Carrieri CG, Carrieri GG. O Estado frente à temática das drogas lícitas e ilícitas: avanços da nova legislação e desafios frente ao Sistema Único de Saúde. SMAD. 2008 jul-dez;4(2):1-13.

5. Meloni JN, Laranjeira R. Custo Social e de saúde do consumo do álcool. Rev Bras Psiquiatr. 2004 janfev;26(1): 7-10.

6. Secretaria Nacional Antidrogas (BR). Levantamento Nacional sobre os padrões de consumo de álcool na população brasileira. Brasília: Secretaria Nacional Antidrogas; 2007.

7. Babor TF, Higgins-Biddle JC. Brief intervention for hazardous and harmful drinking: a manual for use in primary care. Geneva: World Health Organization; 2001.

8. Minayo MCS, Assis SG, Souza ER. Avaliação por triangulação de métodos: abordagem de programas sociais. Rio de Janeiro: Editora Fiocruz; 2005. 
9. Karam H. O sujeito entre a alcoolização e a cidadania: perspectiva clínica do trabalho. Rev Psiquiatr RS. 2003 set-dez;25(3):468-74.

10. Ribeiro M. Organização de serviços para o tratamento de dependência do álcool. Rev Bras Psiquiatr 2004;26(Supl 1):59-62.

11. Moretti-Pires RO. Complexidade em Saúde da Família e formação do futuro profissional de saúde. Interface comunic saude educ 2009 (in press).

12. Corradi-Webster CM, Laprega MR, Furtado, EF. Avaliação do desempenho do CAGE com pacientes psiquiátricos ambulatoriais. Rev. Latino-Am. Enfermagem. 2005;13(2):1213-8.

13. Vargas D, Luis MAV. Alcohol, alcoholism and alcohol addicts: conceptions and attitudes of nurses from district basic health centers. Rev. Latino-Am. Enfermagem. 2008;16(no.spe):543-50.

14. Vargas D, Luis MAV. Development and validation of a scale of attitudes towards alcohol, alcoholism and alcoholics. Rev. Latino-Am. Enfermagem. 2008;16(5):895-902.

15. Ronzani TM, Ribeiro MS, Amaral MB, Formigoni MLO. Implantação de rotinas de rastreamento do uso de risco de álcool e de uma intervenção breve na atenção primária à saúde: dificuldades a serem superadas. Cad Saúde Pública. 2005 mai-jun;21(3):852-61.

16. Corradi-Webster CM, Minto EC, Aquino F, Abade F, Yosetake LL, Gorayeb R, et AL. Capacitação de profissionais de Programa de Saúde da Família em estratégias de diagnóstico e intervenções breves para o uso problemático de álcool. SMAD. 2005 jan-jun;1(1):1-10.

17. Furtado EF, Corradi-Webster CM, Laprega MR. Implementing brief interventions for alcohol problems in the public health system in the region of Ribeirão Preto, Brazil: evaluation of the PAI-PAD training model. Nordic Stud Alcohol Drugs. 2008;25(1):539-51.

18. Aalto M, Pekuri P, Seppa K. Primary health care nurses' and physicians' attitudes, knowledge and beliefs regarding brief intervention for heavy drinkers. Addiction. 2001;96:305-11.

19. Marques ACPR, Furtado EF. Intervenções breves para problemas relacionados ao álcool. Rev Bras Psiquiatr. 2004;26(Supl I):28-32.

20. Minto EC, Corradi-Webster CM, Gorayeb R, Laprega MR, Furtado EF. Intervenções breves para o uso abusivo de álcool na atenção primária. Epidemiol Serv Saúde. 2007 jul-set;16(3):207-20. 